\title{
Extended-POD based acoustic analysis of separated aerofoils via simultaneous time-resolved PIV and force measurements
}

\author{
D. W. Carter $^{1 *}$, M. Ferreira ${ }^{1}$, B. Ganapathisubramani ${ }^{1}$ \\ ${ }^{1}$ University of Southampton, Dept of Aeronautical \& Astronautical Engineering, Southampton, United \\ Kingdom \\ *D.W.Carter@soton.ac.uk
}

\begin{abstract}
We present a cross-correlation based analysis of the acoustic field generated in the wake of NACA 0012 and NACA 65410 aerofoils at a chord-based Reynolds number $R e_{c}=75000$ as obtained from pressure fields reconstructed from a series of planar time-resolved Particle Image Velocimetry (PIV) experiments. The experiments are performed in a water channel facility located at the University of Southampton with an overhead carriage system to allow precise control of the angle of attack $\alpha$ of the aerofoils as well as to mount them to a six-axis force/torque transducer to allow for simultaneous load measurements. Angles of attack in the range $4^{\circ}<\alpha<17^{\circ}$ are explored corresponding to a range of conditions from zero flow separation to fully stalled.

A high-speed Nd:YLF laser is directed in from either side of the facility to simultaneously illuminate the pressure, suction, and trailing regions of the aerofoil and three 4 megapixel high-speed Phantom Veo 640-S cameras are synchronized to capture the entire flow surrounding the aerofoil. PIV post-processing is performed on the individual fields of view separately and then stitched using a calibration reference (fig 1 (a)). The instantaneous planar pressure fields are reconstructed from the stitched time-resolved velocity fields through the application of Taylor's hypothesis (de Kat and Ganapathisubramani. 2012) and Dirichlet boundary conditions approximated from the free stream using Bernoulli's equation. The pressure reconstruction is assessed using the NACA 0012 case at low-angle of attack $\alpha=4^{\circ}$ and is shown to provide reasonable mean surface pressure distribution as compared to a dynamically matched RANS simulation employing a k- $\omega$ SST model (fig $1 \mathrm{p}, \mathrm{c}$ ). By altering the domain size of the RANS simulation, it was determined that the limited domain size of the stitched PIV adversely impacts the accuracy of the pressure reconstruction particularly near the leading edge where the gradients are largest.

The far-field acoustics emanating from the aerofoil surface are approximated from the pressure fields through the application of Curle's analogy (Koschatzky et al., 2011). The present study focuses on the dipole noise sources emanating from the surface of the aerofoil. Motivated by the relationship between vorticity and sound production (Powell, 1995), the extended Proper Orthogonal Decomposition (EPOD, Borée, 2003) was used to reconstruct the pressure and unsteady pressure fields that are most correlated with three quantities: the out-of-plane vorticity, the partial unsteady lamb vector, and the forces and moments experienced by the foil. These correlated reconstructed fields are then applied to Curle's analogy in order to investigate far-field acoustic features that result.

We find the resulting far-field acoustics obtained from the correlation-based pressure reconstructions are found to be more closely related to the temporal behavior of the vorticity fields compared to the unsteady partial lamb vector and the forces and moments based on their overall sound pressure level. The directivity however is not significantly impacted by the correlating quantity, suggesting that the propagation direction is primarily driven by the geometry of the aerofoil.
\end{abstract}

\section{Acknowledgements}

We gratefully acknowledge financial support from EU H2020 project HOMER (Grant Ref No: 769237) and EPSRC (Grant Ref No : EP/R010900/1). 

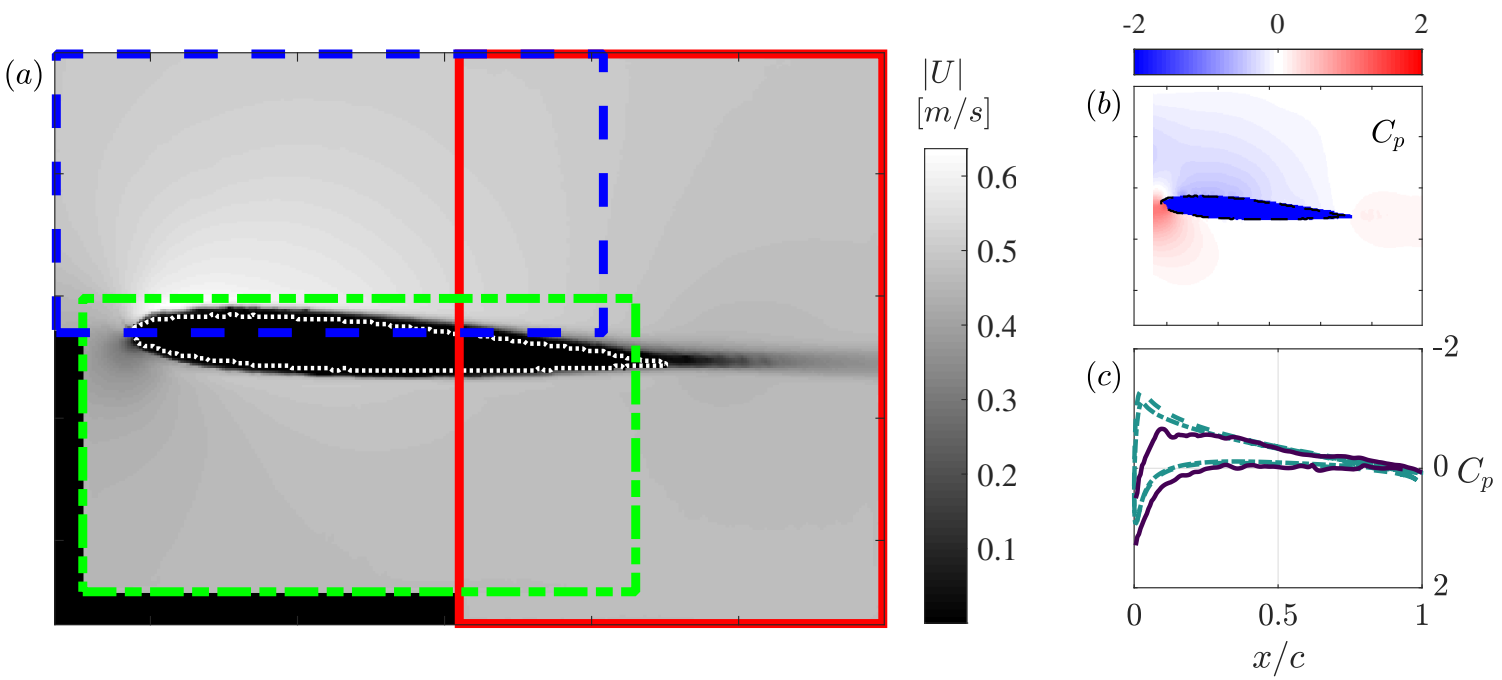

Figure 1: Pseudo-color of mean velocity magnitude from the stitched PIV for the NACA 0012 at $\alpha=4^{\circ}$ (a). The suction, pressure, and trailing regions corresponding to the individual PIV fields of view are shown with dashed, dashed-dot, and solid rectangles respectively. Pseudo color of the mean pressure coefficient field is shown in (b) with corresponding surface pressure distribution in (c) from the PIV (solid) and from dynamically matched RANS model (dashed).

\section{References}

Borée J (2003) Extended proper orthogonal decomposition: a tool to analyse correlated events in turbulent flows. Experiments in fluids 35:188-192

de Kat R and Ganapathisubramani B (2012) Pressure from particle image velocimetry for convective flows: a taylor's hypothesis approach. Measurement Science and Technology 24:024002

Koschatzky V, Moore P, Westerweel J, Scarano F, and Boersma B (2011) High speed piv applied to aerodynamic noise investigation. Experiments in fluids 50:863-876

Powell A (1995) Vortex sound theory: Direct proof of equivalence of "vortex force"and "vorticityalone"formulations. The Journal of the Acoustical Society of America 97:1534-1537 